\title{
Rapid anticonvulsant monitoring in an epilepsy clinic
}

\author{
P N Houtman, S. K Hall, A Green, G W Rylance
}

\begin{abstract}
The relevance of providing a rapid anticonvulsant monitoring service was assessed over a five year period at a paediatric epilepsy outpatient clinic. Altogether 481 drug assays were performed on 144 patients when considered clinically indicated. Drugs most frequently assayed were carbamazepine and sodium valproate, singly or in combination; $\mathbf{9 0 \%}$ of assays performed for phenytoin were from patients who were also taking another anticonvulsant. There were only six assays for ethosuximide and $\mathbf{1 0}$ for phenobarbitone.

Physician's choice of drug dosage was recorded on a questionnaire before and after each assay result was known. Comprehensive patient details were analysed by a paediatric clinical pharmacologist, whose decision as to total daily anticonvulsant dosage was affected by knowledge of the drug concentration significantly less often than that of the clinicians for all the more commonly assayed drugs.

There were a large number of drug assays that had no discernable clinical application. A more discriminating use of assays may both improve patient management and reduce considerably the number of anticonvulsant assays required.
\end{abstract}

Monitoring of anticonvulsant drugs is considered an essential part of the management of epilepsy. ${ }^{12}$ Confidence in the concept that attending to the plasma concentrations of these drugs is the main factor in treating most patients has lessened, however, and this is true in paediatric as well as adult practice. ${ }^{3-5}$

Anticonvulsant monitoring is considered relevant to several areas of management. Firstly is it useful in those patients receiving drugs such as phenytoin or multiple treatment regimens in whom dose related toxicity or poor seizure control necessitates dosage adjustment. Secondly, it can be used as an aid in the assessment of toxicity particularly in those with mental or physical handicap, and possibly in young children. Thirdly, it is useful in the presence of systemic disease when drug handling may be affected unpredictably; finally it can be used in checking compliance. ${ }^{36}$

There is usually a delay of several hours or

Children's Hospital, Ladywood Middleway, $P$ N Houtman

$S \mathrm{~K}$ Hall

A Green

G W Rylance

Correspondence to:

Dr Rylance

Accepted 10 October 1989 immediately available clearly has potential advantages, as any relevant changes in drug treatment can be discussed and instituted without need for further consultation, direct or indirect. Apart from these practical advantages it has been difficult to show objectively a major effect on patient management. ${ }^{78}$

This study documents the usefulness of such a service in a paediatric epilepsy clinic, but also assesses the effects on drug usage.

\section{Patients and methods}

The study was conducted over a five year period from 1982 in the specialist epilepsy outpatient clinics at the Children's Hospital, Birmingham. The patients were seen by one of three consultants experienced in the management of paediatric epilepsy.

Those patients in whom drug assays were considered to be clinically indicated had capillary blood samples taken immediately after an initial interview. In each case, the clinician filled a specifically designed request form on which were not only relevant patient details, drug doses, and the time the last dose was given, but also the proposed course of management in the absence of knowledge of drug concentration.

Plasma drug concentrations of carbamazepine, sodium valproate, phenytoin, ethosuximide, and phenobarbitone were measured using the enzyme multiplied immunoassay technique. ${ }^{9}$ The results were available within 20 minutes.

Each patient was seen again and a continuing treatment regimen established using knowledge of the drug concentrations. At this point the clinician completed the form describing the action taken with knowledge of drug concentration.

At the end of the study period, each completed assay request form was transcribed by one investigator (PNH) onto a further form giving patient details as in the clinical request form but excluding the patient's name. In the great majority of cases, however, the patients' clinical records were also investigated so that a more detailed appraisal of disease activity, including a three tier arbitrary scale of severity and any recent trends, could be documented. These forms contained at least as much, and in most cases considerably more, information than would be provided on an assay request form. The other investigator, a paediatric clinical pharmacologist (GWR) examined these forms and in the absence of knowledge of drug concentration documented his proposed drug management strategy. The drug concentration was then added to the form and his opinion given in the light of the drug assay result. The 'investigating pharmacologist' thus emulated 
the clinician in being initially 'blind' to the drug concentration. An attempt at documenting follow up information was made through the perusal of the clinical notes but this assessment was subjective.

\section{Results}

PATIENT AND ASSAY STATISTICS

A total of 349 blood samples was collected from 144 patients ( 79 boys, 65 girls) over a five year period. Sixty five patients were tested at the clinic on more than one occasion during the study. Ages ranged from 1 to 20 years and the median age was 11 years. No patient had significant hepatic or renal disease.

One hundred and two samples (29\%) were from patients who had major motor seizures alone; $88(25 \%)$ were from those with temporal lobe epilepsy; 33 (10\%) with petit mal epilepsy, and $32(9 \%)$ with partial seizures. Twenty one samples $(6 \%)$ were taken on those with myoclonic jerks and $18(5 \%)$ on those with febrile convulsions. A further $55(16 \%)$ had a mixed epilepsy picture. The above classification into types of epilepsy is not necessarily standardised, and takes into account the nomenclature used by the consultants responsible for the cases.

A total of 481 assays was performed. Figure 1 shows the distribution of samples with respect to drugs assayed. Thus $238(68 \%)$ samples were assayed for carbamazepine and 180 (52\%) for sodium valproate. About half of these samples were assayed for two anticonvulsants. A total of

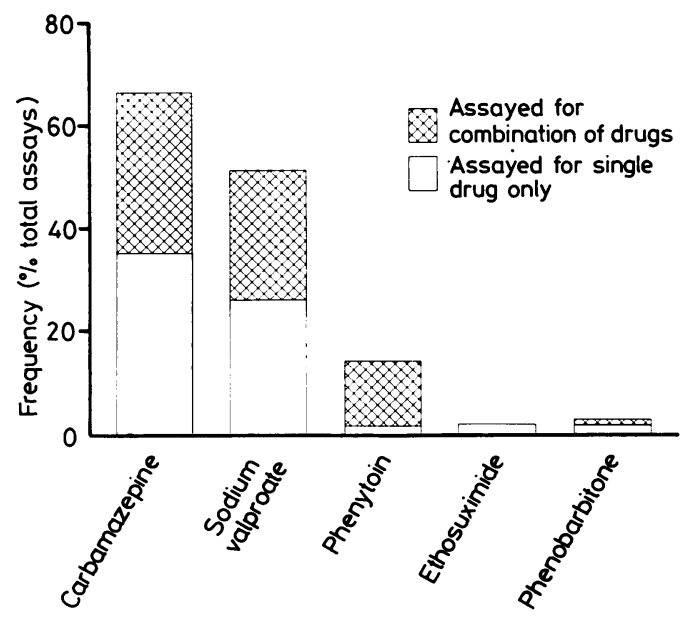

Figure I Number of assays performed for each drug as a percentage of total assays.
229 samples $(66 \%)$ were assayed for only one drug, 74 (21\%) for carbamazepine and sodium valproate, $35(10 \%)$ for other combinations of two drugs, and 11 (3\%) for three drugs (carbamazepine, sodium valproate, and phenytoin in all cases). Of samples processed for phenytoin assay, 42 patients (90\%) were also taking another anticonvulsant. In contrast, in the small number of ethosuximide assays performed, no patient was on another anticonvulsant.

\section{DISTRIBUTION OF PLASMA CONCENTRATIONS}

The distributions of plasma concentrations for carbamazepine, sodium valproate, and phenytoin are shown in fig 2 . For the other drugs the numbers were small; in the case of ethosuximide the maximum plasma concentration was $62 \mathrm{mg} / \mathrm{l}(440 \mu \mathrm{mol} / \mathrm{l})$ and for phenobarbitone it was $28 \mathrm{mg} / \mathrm{l}(120 \mu \mathrm{mol} / \mathrm{l})$.

\section{COMPLIANCE}

Referring to patient notes, it was possible to identify 18 incidents in which compliance was considered to be a significant factor in failure of drug management. Of these, only five assays could be said to have contributed to the assessment of non-compliance by being less than that expected by the clinician. In six patients, however, there was some degree of communication problem involving drug management to the extent that the patient did not receive the drug regimen considered applicable. There was also a high proportion of cases (at least $10 \%$ ) in which parents themselves would adjust the anticonvulsant dose between clinic appointments, depending on clinical features.

\section{TOXICITY}

For carbamazepine there were eight documented cases of significant side effects. Three of these were transient involving lethargy on starting the drug. The others involved visual disturbances and in one case there was a possible related change in behaviour. The carbamazepine concentration was 12-16 mg/l (50-68 $\mu \mathrm{mol} / \mathrm{l})$ in all cases of suppossed toxicity. For sodium valproate there was one case of nausea and three cases of sparse or curly hair growth. There was only one case of documented phenytoin toxicity, that of ataxia (phenytoin concentration $22 \cdot 3 \mathrm{mg} / \mathrm{l}(88 \mu \mathrm{mol} / \mathrm{l}))$, and no cases of ethosuximide or phenobarbitone toxicity.

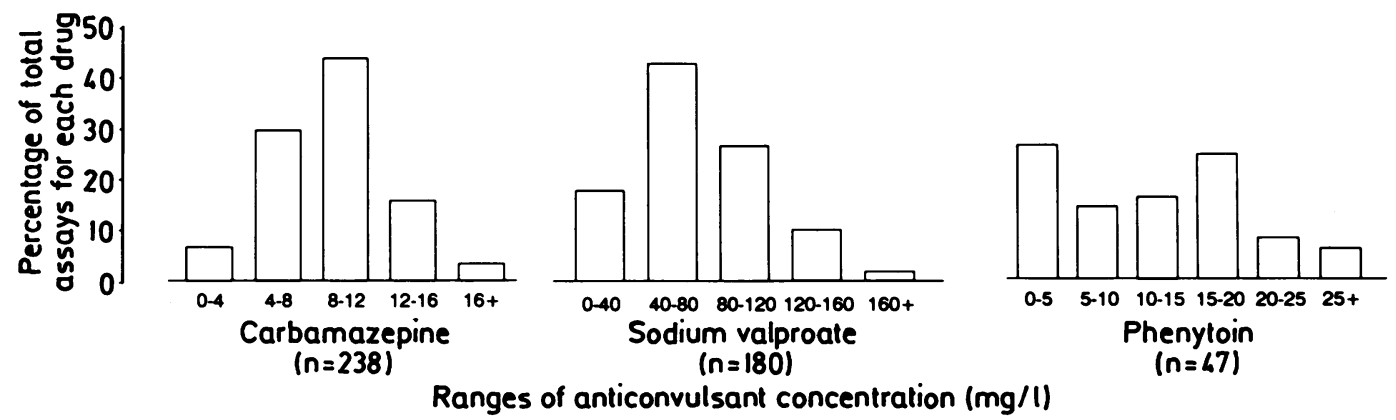

Figure 2 Distribution of assays in relation to concentration ranges for carbamazepine, sodium valproate, and phenytoin. To convert $\mathrm{mg} / \mathrm{l}$ to $\mu \mathrm{mol} / \mathrm{l}$ : carbamazepine, $\times 4 \cdot 2$; sodium valproate, $\times 6 \cdot 9$; and phenytoin, $\times 4 \cdot 0$. 
DRUG DOSE-CONCENTRATION ASSOCIATIONS There were positive linear correlations between the weight related dose and drug concentration for carbamazepine and sodium valproate $(\mathrm{r}=0.46$ and $\mathrm{r}=0.36$ respectively; $\mathrm{p}<0.001)$. For phenytoin a positive association was shown when subdividing into plots of individual patients who had more than two drug assays, as exemplified in fig 3 .

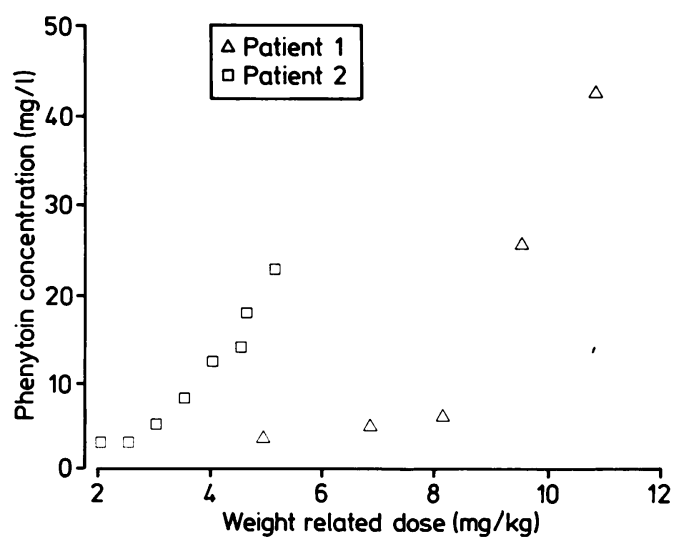

Figure 3 Association between weight related dose and drug concentration for phenytoin in two patients. To convert mg/l to umolll: phenytoin, $\times 4 \cdot 0$.
EFFECT OF DRUG CONCENTRATION ON PATIENT MANAGEMENT

Action of clinician

Table 1 shows the action taken by the clinicians in terms of changes made to total daily anticonvulsant dose as a percentage of the total number of assays for each drug. For carbamazepine a proposed decision by the clinician to change the total daily dose before knowledge of drug concentration was made in $19 \%$ of cases, and this proportion was slightly more with knowledge of the concentration. For sodium valproate and phenytoin the initial figure was approximately $30 \%$ of cases, and again there was an increase in the number of total daily dose changes made after knowledge of the drug concentration. The low numbers for ethosuximide and phenobarbitone prevent any meaningful analysis of the data.

Table 1 also shows the proportion of cases in which there was any change in decision making by the clinician from that before to that after knowledge of the drug concentration; this includes those cases where an initial decision to change dosage was cancelled or reversed on knowledge of the level.

Figure 4 shows the percentage of drug doses changed for carbamazepine, sodium valproate, and phenytoin in relation to the concentration range in which the result fell.

Table 1 Clinician: changes of treatment and decision

\begin{tabular}{|c|c|c|c|}
\hline \multirow[t]{2}{*}{ Drug } & \multicolumn{2}{|l|}{$\begin{array}{l}\text { Change of treatment } \\
(\% \text { of total })\end{array}$} & \multirow[t]{2}{*}{$\begin{array}{l}\text { Change of decision } \\
(\% \text { of total })\end{array}$} \\
\hline & Before knowledge & After knowledge & \\
\hline $\begin{array}{l}\text { Carbamazepine }(n=238) \\
\text { Sodium valproate }(n=180) \\
\text { Phenytoin }(n=47) \\
\text { Ethosuximide }(n=6) \\
\text { Phenobarbitone }(n=10)\end{array}$ & $\begin{array}{c}19 \\
29 \\
32 \\
(17) \\
(10)\end{array}$ & $\begin{array}{c}23 \\
36 \\
51 \\
(17) \\
(20)\end{array}$ & $\begin{array}{c}14 \\
22 \\
34 \\
(0) \\
(10)\end{array}$ \\
\hline
\end{tabular}

Figures in parentheses denote small sample numbers.
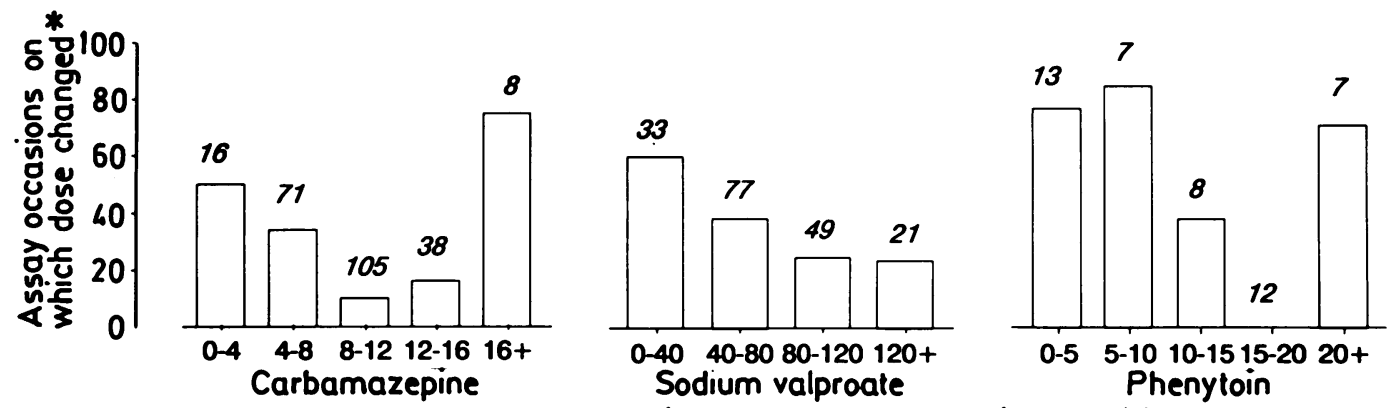

Ranges of anticonvulsant concentration (mg/l)

Figure 4 Occasions on which dose changed by clinician in relation to concentration ranges for carbamazepine, sodium valproate, and phenytoin. The numbers are the total within the concentration range; $*$ of total within range. To convert $\mathrm{mg} / \mathrm{l}$ to $\mu \mathrm{mol} / \mathrm{l}$ : carbamazepine, $\times 4 \cdot 2$; sodium valproate, $\times 6 \cdot 9$; and phenytoin, $\times 4 \cdot 0$.

Table 2 Pharmacologist: changes of treatment and decision

\begin{tabular}{llcc}
\hline Drug & $\begin{array}{l}\text { Change of treatment } \\
\text { (\% of total) }\end{array}$ & After knowledge & $\begin{array}{l}\text { Change of decision } \\
\text { (\% of total) }\end{array}$ \\
\cline { 2 - 4 } Before knowledge & 57 & 57 &. \\
\hline Carbamazepine $(\mathrm{n}=238)$ & 49 & 49 & 8 \\
Sodium valproate $(\mathrm{n}=180)$ & 70 & 80 & 12 \\
Phenytoin ( $=47)$ & $(17)$ & $(33)$ & 23 \\
Ethosuximide $(\mathrm{n}=6)$ & $(40)$ & $(70)$ & $(17)$ \\
Phenobarbitone $(\mathrm{n}=10)$ & & $(30)$ & \\
\hline
\end{tabular}

Figures in parentheses denote small sample numbers. 


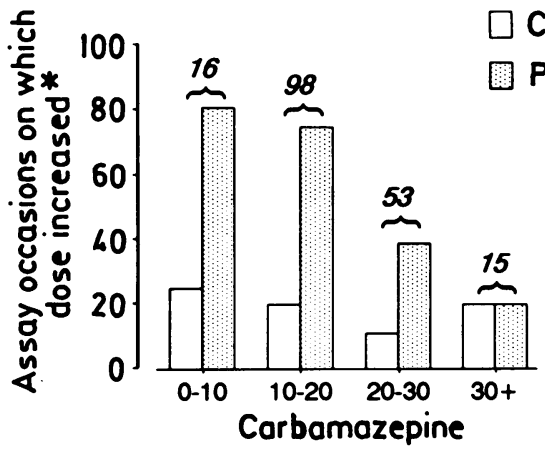

Clinicion

Pharmacologist
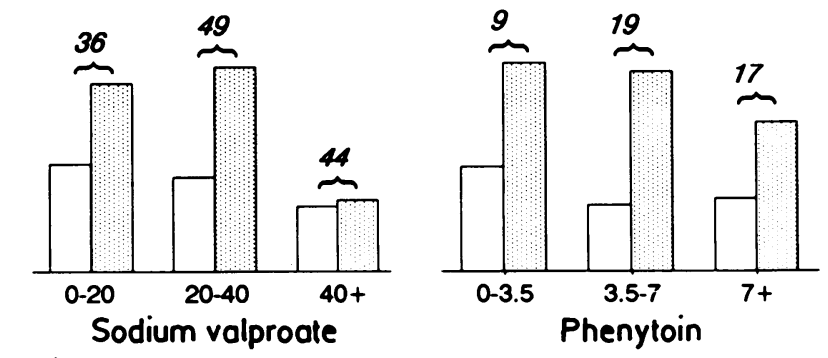

Current anticonvulsant dosage range $(\mathrm{mg} / \mathrm{kg})$

Figure 5 Occasions on which dose increased in relation to current dosage range for carbamazepine, sodium valproate, and phenytoin. The numbers are the total within dosage range; $* \%$ of total within range.

Actions of pharmacologist compared with clinician Table 2 shows the actions proposed by the paediatric pharmacologist, and these results relate to table 1 for the clinician.

There were considerable differences between the actions of the clinicians and those of the paediatric pharmacologist. In approximately half of all assays the proposed course of action differed between the two, both before and after knowledge of drug concentration. Figure 5 compares the actions of clinician and pharmacologist with respect to weight related total daily dose before drug concentration was known. In this figure only those patients whose investigation forms had led to a designation of moderately severe or severe epilepsy are included, as patients with mild epilepsy might be considered to be well enough controlled even if receiving low doses of anticonvulsants. The pharmacologist was considerably more likely to increase the dose throughout the range of doses but particularly so at the lower end of the dosage range.

\section{Discussion}

Knowledge of anticonvulsant plasma concentration affected the clinicians' treatment plans in only a small proportion of cases. Although these findings alone may suggest that therapeutic drug monitoring was simply a wasted exercise in the vast majority of cases, the only direct measure of the usefulness of such assays would be to assess clinical effectiveness in some way. Such a global study would be unlikely to succeed because, even with a relatively large sample size as in this study, there are too many variables of morbidity in the population of children with epilepsy as a whole, and with regard to time in an individual patient, for such a study to be objective. ${ }^{10}$ Instead this study provides information about some of the factors involved in monitoring of anticonvulsant concentration, and is of value to the clinician in evaluating the need for an assay result in an individual case.

The changes in decision making by the clinicians were not evenly spread between the anticonvulsant drugs. We have shown that children exhibit saturation kinetics well within the clinical range of plasma concentrations for phenytoin (fig 3). ${ }^{11} 12$ This would be a contribu- tory factor in accounting for the large number of samples where the plasma concentration was barely detectable and also the significant number well above the recognised therapeutic range of plasma concentrations. This unpredictability of the plasma concentration would also explain the considerably increased number of changes of decision after knowledge of the concentration made by the clinician for phenytoin over other anticonvulsants. In contrast, for carbamazepine there were few changes of decision and as might be expected, the plasma concentrations of the assays formed a fairly symmetrical distribution around the recognised therapeutic range indicating a high level of predictability. It has recently been reported in adult practice that changes to treatment dosage are made throughout the range of drug concentrations (MJ Brodie. Abstract presented at 4th biannual British/Danish/Dutch epilepsy congress, Amsterdam, September 1988) but, in this study and for carbamazepine at least, there was a strong tendency for the clinician not to change dosage when the drug concentration was in the middle of the therapeutic range (fig 4).

For sodium valproate there appears to be some confidence in a lower limit for therapeutic range but there is no evidence from these data that there is a well defined upper limit for desirable drug concentration. This supports the current view that plasma concentrations of sodium valproate are a poor predictor of efficacy. ${ }^{1314}$ The relevance of plasma monitoring for valproate is further put into question when it is considered that although we found that there was a highly significant correlation between the weight related dose and plasma concentration, the wide scatter of results meant that only just over $10 \%$ of this association could be accounted for by a linear correlation.

Comparison of the clinicians' and the paediatric pharmacologist's approach showed considerable differences. Tables 1 and 2 show that the pharmacologist was likely to change drug dosage significantly more often than clinicians for all the more commonly assayed drugs. But in terms of changes in decision making in the light of knowledge of drug concentration, the pharmacologist made even fewer decision changes than the clinician. In looking for an explanation for these differences there were obviously factors which could not be controlled 
for, particularly the fact that the clinicians actually saw the patients and their families so that decisions were based on relationships between doctor and patient and these could not be transcribed to a written form. Further scrutiny of the results, however, suggests that much of this difference might be accounted for by the pharmacologist tending to increase dosage proportionally more than the clinicians at the lower end of the weight related dosage range. Only those patients having repeated fits are included in this assessment, as those patients free of fits must be considered to be well controlled. Figure 5 confirms that the pharmacologist was considerably more likely to increase the dose throughout the range of dosages but particularly so at the lower dosages. In other words the pharmacologist seemed more aware of the concept of achieving a more appropriate weight related dose than the clinician.

As most assays were performed when the clinician had little apparent intention of changing the drug dosage regimen even without knowledge of drug concentration, it might be supposed that these assays were being done to assess toxicity or compliance. It is perhaps surprising that although there were many phenytoin concentrations well above the recognised plasma therapeutic range, there was only one case of documented toxicity for this drug. ${ }^{15}$ Toxicity therefore must have had little effect on the proportion of decision changes. Although compliance is accepted to be a major factor in problems of management, this study showed that in only a small number of cases was the discovery or the assessment of the non-compliance significantly attributable to the assays.

The epidemiological data in this study can have only indirect relevance to the population of children with epilepsy as a whole. For example, in assessing toxicity, the data are of value only for dose related events, and so give no information about the incidence of such idiosyncratic reactions as carbamazepine induced rashes. Similarly the data on the type of epilepsy are more reflective of the apparent need for anticonvulsant monitoring than the distribution of epilepsies in childhood. Compared with carbamazepine and sodium valproate, only a small minority of phenytoin assay samples were from patients not taking another anticonvulsant, so that phenytoin appears to be a 'second line' drug in paediatric practice.

A rapid drug monitoring service is expensive, and in this case the cost was at least double that of a conventional drug monitoring service. The cost is in part proportional to the number of assays performed at each clinic session. The advantage of rapid monitoring to this type of study, however, is that it provides the oppor- tunity for the clinician to document a judgment on drug treatment strategy before and after knowledge of the drug concentration without allowing the passage of time to reduce the power of the comparability between the two decisions. A rapid assay service must also be directly advantageous to patient management. In the area of compliance this study has shown that patients do not always receive the intended continuing dosage regimen even with the rapid assay service. Taking into account the practical advantages of communicating directly with the patient, the efficiency of the service cannot be measured merely by comparing basic costs. As in no case did the clinician make any change in decision after knowing the drug concentration for ethosuximide, however, the cost of this drug assay may not be justified.

In conclusion, this study has shown that a large proportion of drug measurements have no discernable clinical application. Differences between the pharmacological characteristics of individual anticonvulsant drugs have been shown but the clinician often does not use the data provided appropriately. A more discriminating use of data from assays may both improve patient management and cut down considerably on the number of anticonvulsant assays required.

1 MacDonald JT. Childhood seizures. Postgrad Med 1988; 83:79-92.

2 Mucklow JC. Therapeutic drug monitoring: can it improve the treatment of epilepsy? F Clin Hosp Pharm 1982;7: $79-85$.

3 Chadwick DW. Overuse of monitoring of blood concentrations of antiepileptic drugs. Br Med $\mathcal{F}$ 1987;294:723-4.

4 Aicardi J. Clinical approach to the management of intractable epilepsy. Dev Med Child Neurol 1988;30:429-40.

5 Shinnar S. Antiepileptic drugs in adolescents. I Adolesc Health Care 1987;8:105-12

6 Bartels H. Rational usage of therapeutic drug monitoring in antiepileptic treatment. Eur F Pediatr 1980;133:193-9.

7 Patsalos PN, Sander JWAS, Oxley J, Lascelles PT. Immediate anticonvulsive drug monitoring in management of epilepsy. Lancet 1987;ii:39.

8 Marty J, Fullinfaw R, Tuckett R, Trembath P, King J. Evaluation of the provision of rapid drug plasma assays in an outpatient anticonvulsant clinic. Ther Drug Monit 1981; 3:253-8.

9 Oellerich $M$. Enzyme immunoassays in clinical chemistry: present status and trends. F Clin Chem Clin Biochem 1980; 18:197-208.

10 Froescher W, Eichelbaum M, Gugler R, Hildenbrand G Penin H. Report on a controlled trial on the significance of plasma antiepileptic drug monitoring. In. Johannessen SI, Schmidt D, Meinardi $\mathrm{H}$, eds. Antiepileptic therapy: advances in drug monitoring. New York: Raven Press, 1980:263-70.

11 Richens A. Clinical pharmacokinetics of phenytoin. Clin Pharmacokinet 1979;4:153-69.

12 Rylance G. Drug disposition in infancy and childhood. In: Parsonage M, Grant RHE, Craig AG, Ward AA, eds. Advances in epileptology: XIVth epilepsy international symposium. New York: Raven Press, 1983:273-82.

13 Chadwick DW. Concentration-effect relationships of valproic acid. Clin Pharmacokinet 1985;10:155-63.

14 Armour DJ, Veitch GBA. Is valproate monotherapy a practical possibility in chronically uncontrolled epilepsy? $\mathcal{F} \mathrm{Clin}$ Pharm Ther 1988;13:53-64.

15 Schmidt D, Lempert T. Phenytoin toxicity: relation to plasma concentration. In: Wolf $\mathrm{P}$, Dam $M$, Janz $\mathrm{D}$, Driefuss FE, eds. Advances in epileptology. Vol 16. New York: Raven Press, 1987:449-52. 